\title{
Germanica
}

GERMANICA $\quad 38 \mid 2006$

Voix étrangères en langue allemande

\section{Said Bewegung bis zur Ent-Fremdung Ein Portrait}

Une dynamique en quête de soi

\section{Francine Rouby}

\section{(2) OpenEdition}

Journals

Édition électronique

URL : http://journals.openedition.org/germanica/412

DOI : 10.4000/germanica.412

ISSN : 2107-0784

\section{Éditeur}

Université de Lille

\section{Édition imprimée}

Date de publication : 1 juin 2006

Pagination : 157-171

ISBN : 2-913857-17-5

ISSN : 0984-2632

\section{Référence électronique}

Francine Rouby, « Said Bewegung bis zur Ent-Fremdung Ein Portrait », Germanica [Online], 38 | 2006,

Online erschienen am: 19 Februar 2010, abgerufen am 06 Oktober 2020. URL : http://

journals.openedition.org/germanica/412 ; DOI : https://doi.org/10.4000/germanica.412

Ce document a été généré automatiquement le 6 octobre 2020.

(c) Tous droits réservés 


\title{
Said Bewegung bis zur Ent- Fremdung Ein Portrait
}

Une dynamique en quête de soi

\author{
Francine Rouby
}

I.

1 SAID (Pseudonym) wurde 1947 in Teheran geboren. Im Jahre 1965 kam er nach Deutschland, nicht als Exilant, sondern - wie damals viele andere Iraner - um zu studieren. Er sollte - es war der Wunsch seines Vaters - Ingenieur werden. Der damals 17 jährige war aber anders begabt, hatte andere Pläne.

2 Im Iran hatte er als Schüler keine politische Tätigkeit gehabt. Außer vielleicht die, dass er verbotene Bücher las.

es waren werke, die in europa überall ganz normal zu kaufen sind, z.b. schwarz und rot von stendhal, die insel der pinguine von anatole france, gorki war verboten, sartre auch. das waren die bücher, die uns interessierten ${ }^{1}$.

Frühjahr 1981 (unveröffentlicht) ${ }^{2}$

In Deutschland begann SAID dann ziemlich schnell politisch aktiv zu werden, und zwar in der Cisnu, der oppositionellen exil-iranischen Studentenorganisation. Der Anstoß war die Studentenrevolte 68 und die Demonstrationen gegen den Schahbesuch. münchen war damals bekannt als eins der zentren der politischen aktivitäten im ausland. es waren sehr viele iraner in der ortsgruppe münchen der cisnu organisiert.

die richtung der cisnu war anti-schah und allgemein links. Ebd.

Nach dem Sturz des Schahs dachte er, er könne wieder in den Iran zurückkehren. Die Reise 1979 nach Teheran stellte sich als eine schwere Enttäuschung heraus. Unter dem Regime der Mullahs war es für Intellektuelle weiterhin unmöglich zu leben. Ein Zurück in die Heimat, ein Neuanfang, war nicht mehr denkbar.

4 SAID lebt seitdem in München, wo er als freier Schriftsteller arbeitet. 
Im Jahre 2004 hat er, nach vierzig Jahren, die deutsche Staatsangehörigkeit erhalten.

\section{II.}

6 Schon 1965, damals kaum erst in Deutschland angekommen, war das Bedürfnis aufgekommen, die andere ihm gänzlich fremde Sprache so schnell wie möglich und so perfekt wie möglich zu beherrschen. Der Weg von der Muttersprache zur fremden Sprache war der Weg in eine andere Welt.

7 SAID hat immer nur auf Deutsch geschrieben.

8 In der fremden Sprache leben müssen, leben wollen, mit ihr umgehen können, bedeutet die Distanz zu den fremden Wörtern überbrücken, denn nichts ist mehr selbstverständlich, nichts fließt mehr von selbst. Man kann nicht mehr sagen und schreiben, was die Muttersprache schnell und bereitwillig anbietet. Alles muss überlegt, zersetzt und analysiert werden, der Benutzer der anderen Sprache stockt, hält inne,wird aber gerade deshalb von der fremden Sprache nicht beherrscht.

es sind zwei flüsse in mir. hier der persische, dort der deutsche; jeder stillt einen anderen durst.

zuweilen tauschen sie die plätze, ohne mir bescheid zu geben.

überhaupt habe ich das gefühl, daß sie sich im laufe der letzten 33 jahre dieser zwangsgemeinschaft selbständig gemacht hab

die wörter von beiden flüssen berühren sich zuweilen, mit einer zärtlichen komplizenschaft.

diese flüsse sind miteinander keineswegs verwandt; sie treffen sich nur in meiner mitte - zu meinem leidwesen.

in dem einen fluß schwimme ich mit, meist ohne bewußt zu sein, daß ich schwimme.

in dem anderen kämpfe ich um jedes wort, um nicht zu ertrinken.

zwischenland sprache (unveröffentlicht)

9 Beide Sprachen, beide Welten, die diese „zwangsgemeinschaft“ bilden, sind die notwendige Reibungsfläche, ohne die es für SAID keine Lyrik gibt.

Der Dichter braucht eine

reibungsfläche; jene außenhaut, die er braucht, um seine innenhaut daran zu reiben.

das gedicht: die spannung zwischen den wörtern, [...] ein bedürfnis nach einem anderen ort, nach einer stummen landschaft, nach einem nötigen zwischenraum ohne abwort gottes. [...] flucht ins unbekannte.

wer, wenn ich schrie (unveröffentlicht)

11 Bei SAID ist Vorsicht Gesetz - nur nicht zu geschwätzig sein. Das Unnötige streichen, das Wesentliche herausfiltern, bis zu ihm vordringen. Mehr ist nur Lüge. SAIDs Stil ist minimalistisch. Er schreibt zusammengeballt. Seine Gedichte werden reduziert auf kurze, höchst präzise Zeilen, auf knappe, prägnante Sätze in der Manier des Haikus. Eine ausgeklügelte, ausgefeilte Sprache. Auch in seiner Prosa ist keine Zeile, kein Wort zuviel. Die Dinge werden nicht direkt aufgegriffen, werden nicht zentral behandelt, kommen nie voll ins Licht; sie werden von der Seite beleuchtet, das Einzelne soll das Ganze andeuten. Er selbst sagt ${ }^{3}$ :

ich verabscheue erklärungen, weil ich weise bin.

der weise sieht die bewegungen zwischen den dingen.

er braucht keine erklärungen.

ich hasse erklärungen, sie sind fast wie lügen, aber nicht so schön. 
Und er schreibt weiter:

da die poesie das ganze sucht, ist sie gezwungen, sich mit details zu befassen: mit versatzstücken, mit abfall, mit pailletten.

das gedicht ernährt sich von marginalien, um dem gesamtbild zu dienen.

wer, wenn ich schrie (unveröffentlicht)

Die Rückkehr nach Teheran, die in Wo ich sterbe, ist meine Fremde geschildert wird, ist ein Meisterwerk dieses Stils. Nichts Überflüssiges, nichts Überschwängliches, keine großen Theorien. Aber sehr viel Emotion durch pointillistische Schilderung von alltäglich Erlebtem. Einfache Details, kleine intime Regungen, Begebenheiten, die man leicht übersehen könnte, machen die Intensität dieses Werkes aus.

Die Haustür ist offen, und der Gang ganz dunkel.

Meine verwestliche Hand

Findet keinen Schalter

Links neben der Tür.

Wo ich sterbe, ist meine Fremde S. 24

12 Die Kraft von SAIDs Sprache liegt auch in der Frische, in der Bildhaftigkeit neuer Assoziationen, überraschender, manchmal surrealistisch anmutender Wortbildungen. Inhalt und Form: „eine Harmonie, die sich zuweilen auch verschieben kann (3).“

$$
\begin{aligned}
& \text { das gedicht } \\
& \text { ein stummer schritt } \\
& \text { entlang der sprache } \\
& \text { bis hin zur tiergrenze } \\
& \text { ein schrei } \\
& \text { der das wort verrät } \\
& \text { (unveröffentlicht) }
\end{aligned}
$$

13 Der Dialog mit einer fremden Sprache bringt Bewegung, bietet neue Richtungen, eine andere Sicht der Dinge, neue Perspektiven, also neue Möglichkeiten, Öffnung auf andere Einsichten. Fremdheit und Distanz lösen Kreativität aus und bewirken einen ganz anderen Umgang mit der neuen Sprache, mehr Möglichkeiten in der Benutzung gewisser Begriffe, einen weniger gehemmten Umgang mit der Wortzusammenstellung, mehr Phantasie.

14 SAIDs Metaphorik ist durchwirkt von Bildern aus der Heimat, dem Iran, die den Leser oft bewegen, manchmal befremden. Die Verbindung zwischen dem Wort und seiner Bedeutung wird ver-rückt und bekommt dadurch eine eigenartige Dynamik. Sie ist für den Leser nicht mehr unmittelbar entzifferbar. Wird also zu einem Spannungsfeld. Ein retardierendes Moment. Aber auch fließende Vieldeutigkeit.

15 Jedes Gedicht lebt an sich, aber seine Schönheit blüht in dem Kontext, in seinem Bezug zu den anderen Gedichten der jeweiligen Bändchen erst ganz auf. Liebesgeschichten werden erzählt, spielerisch manchmal, - ernst, sehr oft. Und es sind immer Vorgänge, die da geschildert werden. Anfang und Ende einer Beziehung, die Hoffnung auf Heimkehr, immer wieder aufwallende Sehnsüchte.

\section{III.}

Und Kritik kommt auf. Auf leichten Füßen. Dem Westen wird ganz diskret einen Spiegel vorgehalten. Damit

die poesie das gedächtis sein kann für künftige generationen. Denn eine schönheit ohne gedächtnis - also eine statische schönheit - ist nur mode. 
Wer, wenn ich schrie (unveröffentlicht)

Die Oberfläche der Industriestaaten wird gelüftet, und der faulende Untergrund kommt zum Vorschein, springt dem Leser ins Gesicht. Denn

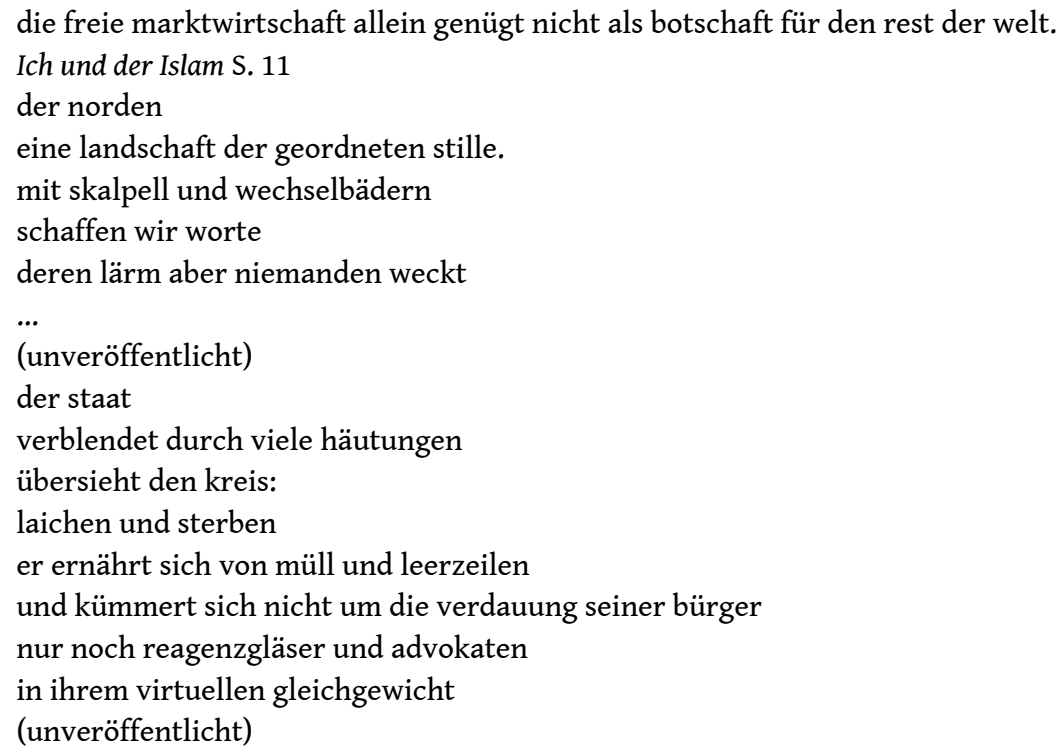

18 Das Fehlverhalten des Westens wird in den polemischen Schriften direkter ausgesprochen, zuweilen auch angeprangert. Denn die Sprache des Dichters ist eine Waffe.

gedichte sind nie unschuldig, der dichter trägt sein gedicht zwischen den zähnen wer, wenn ich schrie (unveröffentlicht)

19 Er schlägt beim Leser hart ein, genau in die Mitte. Er schrieb mir, als ich ihn darauf ansprach:

ich arbeite nach dem prinzip von karate: totale konzentration, dann zuschlagen auf daß der leser fällt (3).

SAID äußert in seiner Kritik niemals ein radikales Denken, will nicht überzeugen, nur warnen. Er will die Augen des Lesers öffnen.

das gedicht ist die radikale verneinung der welt, um irgendwann eine gegenwelt aufzubauen; nein anzudeuten, für künftige ohren.

exil - ein intermezzo (unveröffentlicht)

Das Verhältnis der Intellektuellen zur Politik nimmt SAID als Flüchtling sehr ernst.

... kultur ist eine zu ernste angelegenheit, als dass man sie den politikern oder diktatoren überlässt.

... schriftsteller in unseren ländern - und hier besonders lyriker - [spielen] eine sehr wichtige rolle; da sie zuweilen die freie presse ersetzen müssen. zuweilen sind unsere lyriker in der tat das gewissen der nation. denn sie flüstern in ihren gedichten, wovon die presse laut schweigt...

Brief an Adonis in Ich und der Islam S.162

SAID legt einfach den Finger auf die Wunden unserer Zeit. In den Gedichten wie auch in den politischen Essays und in seinen Fiktionen denunziert er Missstände, Ungerechtigkeit, himmelschreiendes Unrecht, Lieblosigkeit als Folge der Intoleranz schlechthin. Er tut es auch in den Kindergeschichten. Da geht es um einen Teddy, der ein Kind hat, das ihn verstößt, um eine Blume, die sich nicht zu ihrer Farbe bekennen kann, bis sie einen wohlwollenden Freund findet. SAID scheut es aber auch nicht, von Brutalerem zu erzählen, von Folter und Hinrichtung, er schildert Vergewaltigung und 
Willkür. Er gedenkt all derer, die den Freiheitskampf bis zum Schluss ausgekämpft haben, Freunde, Dichter, Politiker und Denker aller Länder, aller Zeiten, die nie aufgegeben und ihr Leben geopfert haben.

Er denunziert die Diktaturen, aber auch den demokratischen Westen, Europa, das seine Versprechungen nicht gehalten hat. Trotz Aufklärung hat Deutschland sechs Millionen Juden ermordet, Frankreich hat mit der Revolution 1789 die Aufklärung auf „das banner der republik geschrieben“ und 1,5 Millionen Algerier ermordet. Um von den Gräueltaten der Engländer in Indien ganz zu schweigen. (Ich und der Islam S. 23)

In der Kritik am Iran des Schahs und dem der Mullahs sind die Fehltritte, die Irrwege der freien Länder herauszulesen.

„gottes ist der orient! gottes ist der okzident!“ diesem satz goethes fügt der ostwestliche flüchtling seinen profanen satz hinzu:

das niemandsland dazwischen ist unseres.

wir können es nur mit liebe befruchten.

Brief an europa (unveröffentlicht)

Der Flüchtling wird auch heute noch mit dem anderen Gesicht Europas konfrontiert.

... ein hässliches [...] schizophrenes europa, mal eine unschuldige jugendliebe, mal eine konvertierbare hure! [...] und der gealterte flüchtling, inzwischen zu einem kompositum geworden aus zwei welten, er liebt und sucht sein europa weiter; und er hofft, dass dieses europa mehr ist als eine finanzschimäre, [...] sondern ein geschenk bleibt, für alle, die freiheit suchen.

Brief an europa (unveröffentlicht)

SAID vertritt die Rückbesinnung auf die eigene iranische Tradition, auf seine Wurzeln. Religion ist für ihn mit Kindheitserinnerungen verbunden, mit Moscheen, die nach Rosenwasser rochen, mit dem Ruf des Muezzin, von dem das Kind Gänsehaut bekommt, mit dem Blut des geschächteten Huhns und der Flagellanten. Er hat die Religion nie ausgeübt und spricht entschieden gegen die Islamische Republik. Er ist vom Pluralismus, der Attraktivität der Demokratie überzeugt. Seine Stellungsnahme ist auch hier beschwichtigend, aufklärerisch und demokratisch. Er sieht die Berührung der Gegensätze als Chance.

... denn die religiosität ist für mich ein viel höherer begriff, als dass ich jetzt

kirchengänger wäre für eine bestimmte kirche oder moschee.

Ich und der Islam S. 42

IV.

Der Schmerz der im Exil Lebenden, der Außenstehenden ist überall und für alle derselbe. Mit sehr viel Humor schreibt er über das Schicksal eines Übersetzers im Iran:

Draußen vor der Tür von Wolfgang Borchert erscheint.

Der Übersetzer ist inzwischen nach Deutschland geflohen.

Nun wartet er selbst draußen vor der Tür.

Der lange Arm der Mullahs S. 64

Und etwas schlechtes Gewissen schimmert ab und zu durch.

und ich saß wieder in meinem sicheren exil,

um täglich vom tod verschiedener freunde zu hören:

hingerichtet, auf der flucht erschossen, zu tode gefoltert.

exil - ein intermezzo (unveröffentlicht)

Er selbst findet sich weder in seiner Heimat, im Iran, noch in Deutschland zurecht. Auch er steht vor der Tür, ist zu einem Ewigen Juden geworden. 
Was ist geschehen mit meiner Stadt?

Der Flieder duftet

nicht wie früher.

Früher gingen die Menschen

einfach langsamer.

Und die Tauben -

sie sitzen nicht mehr auf dem Gehsteig.

Wo ich sterbe, ist meine Fremde S. 67

Ich krieche mühsam hierher

setze mich geräuschvoll hin,

strecke meine Gefühle von mir,

nehme viel Platz ein -

und werde nicht benötigt.

„Brief eines Emigranten“ in Dann schreie ich, bis Stille ist S. 18

durch die jahre des exils wird man ein niemand,

ein geringfügiger fremder «in diesem mühsamen nirgends»,

wie rilke einmal sagte.

nach 28 jahren des exils ist man ein zwischending.

man könnte es auch poetischer ausdrücken,

ein weltbürger, ohne eigenes fenster.

Der lange Arm der Mullahs S. 135

\section{V.}

Die Brücke kann nur durch die Aneignung der fremden Sprache geschlagen werden, eine Notwendigkeit, um zu überleben. Denn die „türen öffneten sich oft erst in dem moment, da man ein klares deutsch sprach...

In Deutschland leben S. 27

In Deutschland begann er,

das iranische terrain zu verlassen, den käfig des exils. ... die isolierung brachte einsamkeit, und da kam die deutsche sprache wie eine art auffanglager.

Ebd. S 30

was mich anbetrifft, ich bin nur ein ungebetener gast,

der als seine eigentliche gastgeberin die deutsche sprache sieht.

man weiss, dass man keine heimat mehr hat.

denn die heimat ist die zeit, die wir verloren haben.

man ist aber bemüht, sich zu trösten, sich zu belügen.

so sagt man sich:

meine eigentliche heimstätte

ist die deutsche sprache, die mir zuflucht geboten hat.

exil - ein intermezzo (unveröffentlicht)

SAID bezeichnet sich gern als "gast und gefangener dieser sprache“. Jedoch, auf das anfängliche Ende jeglicher Vertrautheit folgt eine Dynamik, die den Freiraum ausfüllt:

Erst die geborgenheit der fremden sprache - jenseits der schwülen umarmungen der „muttersprache“ - ermöglicht es dem dichter, seine ängste zu formulieren.

wer, wenn ich schrie (unveröffentlicht)

Er erlangt eine Authentizität durch die Aufrichtigkeit, zu der ihn die fremde Sprache, die deutsche Sprache, zwingt. Eine Mauserung, denn der Exilant muss sich neu definieren, zu sich selbst kommen, trotz Ortverschiebung. Vielleicht sogar besser und intensiver dank Ortverschiebung. Dadurch dass alles neu benannt werden muss, ist das Fremde auch immer Selbstentfremdung.

...gedichte, um herauszubekommen, wohin es mit ihm will 
er tritt eine reise an, deren ziel er nicht kennt.

exil - ein intermezzo (unveröffentlicht)

Mit dieser Erkenntnis legt er den Finger auf seine eigenen Wunden. Die Sprache wird zur Brücke zwischen zwei Kulturen, zwischen Orient und Okzident, zur Brücke zu dem eigenen Ich. Die eigentliche Suche, die unternommen wird, ist die Suche nach sich selbst ...

\section{In Gedichten}

habe ich mich immer entwirrt, und sie verwirren die anderen, so sind Gedichte.

Selbstbildnis für eine ferne Mutter S. 38

... in der Kindheit.

gedichte in einer fremden sprache, um bruchstücke einer identität zu sammeln... gegen das vergessen ... schmale brücke zur kindheit ... wo die flucht zu ende ist? exil - ein intermezzo (unveröffentlicht)

Heimatsuche in der iranischen Kindheit, an die man sich festklammert, eine rückwärtsgewandte Weltanschauung, die verwirrt und die Gegenwart betrübt.

Ein innenhof, im süden, unter blauem himmel ... - ein flughafen im norden, das kind - 15 jahre später - in der hand einen großen koffer ... zwischen diesen bildern - jedes auf seine weise verzerrt - taumelt noch immer das kind, seit nunmehr 35 jahren und kann sich nicht entscheiden für keines der bilder: das erste ist weit entrückt und dennoch vertraut, das zweite nah und dennoch fremd.

exil - ein intermezzo (unveröffentlicht)

Die Kindheit ist für SAID, wie für viele Asylanten und Exilanten, die einzige Bindung zu der Vergangenheit. Kindheitserinnerungen, meistens Erlebnisse aus der ganz frühen Kindheit, kommen immer wieder, als Schutz vor der Kälte des Gastlandes, auch als Mittel, die neue Heimat zu sehen, zu verstehen, das neue an dem alten zu messen. Die Kindheit, die Herkunft, ist das einzige, was man ja noch wirklich besitzt, je besessen hat.

Weg von meinem Namen,

dem einzigen

was ich wirklich besitze.

Selbstbildnis für eine ferne Mutter S. 17

Eine Kindheit, die in SAIDs Lebensgeschichte auch Schmerz bedeutete. Er hat sie nicht verbrämt. Eine Kindheit ohne Mutter, eine einsame Kindheit. Am Schönsten schildert er diesen Schmerz, diese Sehnsucht nach Mutterliebe, nach Wärme in seinen Kindergeschichten. In einer schlichten, einfachen aber höchst sensiblen Sprache, meisterhaft inszeniert, machen sich die Blume, die keine Farbe hatte, und Felix, der kleine kuschelige Teddybär (unveröffentlicht) auf den Weg, auf die Suche nach Halt und Bestand, einem Ort, wo sie sich wohl fühlen dürfen. Die Parallelen zum Exil werden immer wieder betont.

das kind will nur begreifen, will licht und bewegung

das kind schreibt gedichte, sucht, hofft und dankt seiner fremden sprache.

das kind bewegt sich fort.

bis es ein saurier wird - ein weltbürger ohne eigenes fenster.

exil - ein intermezzo (unveröffentlicht)

SAID inszeniert sich gerne als Kind.

Denn der Exilierte,

Mutter,

ist ein Kind, 
das jeder schlägt;

es wird mürbe,

aber nicht reif.“

Selbstbildnis für eine ferne Mutter S. 13

VI.

31 Mit den Augen des Kindes, das er war, sieht und erlebt er die Gegenwart. Kindheit ist trotz allem das verlassene Paradies. Ist SAID dadurch in seinen rückwärtsgewandten Träumen stehen geblieben? Dies bedeutete aber Perspektivlosigkeit. Äußere Grenze, innere Grenze...

Keine Dynamik? Oder eine im Kreis sich drehende Dynamik? Die Heimat wurde nicht ersetzt, die Erinnerung an sie ist als Fiktion verblasst. Die Vergangenheit ist zum Traum der Gegenwart geworden. Der Verlust hat sich verewigt.

\section{Beide Welten kann der Exilant nicht in Einklang bringen, die Befremdung bleibt.}

In der Heimat, im Iran, folgt Enttäuschung auf die Sehnsucht...

wie gütig

die passanten sind -

sie sprechen persisch!

Wo ich sterbe, ist meine Fremde S.18

welch eine freude!

hier schreibt man meinen namen,

ohne dass ich ihn buchstabieren muss.

Ebd. S.21

In der Heimat

Immer wieder

falle ich den Menschen auf -

Als Fremder.

Woran liegt es?

An meinem Blick?

An meiner ungebügelten Hose?

Weil ich den Zeitungsverkäufer zuerst grüße?

Weil ich auch Frauen die Hand schütteln will?

Sind wir -

Die Heimkehrer -

Eine eigene Rasse geworden?"

Ebd. S. 71

... und in Deutschland kommt es auch nicht zu neuen Wurzeln.

11. august 1993

heute fragt mich ein ganz normaler deutscher auf dem marienplatz nach einer adresse. kein alternatives junges paar, das nur seine solidarität bekunden will. kein rüstiger rentner, der sich und mir beweisen will, dass er nichts gegen ausländer hat; sondern ein ganz normaler bürger.

Notizen (unveröffentlicht)

Nach einer Japanreise überkommt ihn ein seltsames Gefühl:

ich will hier gar nicht weg. hier war ich auch ein fremder. aber die totale fremdheit, begleitet vom seltenen erlebnis der sprachlosigkeit, nimmt den druck weg. hier habe ich die fremdheit nicht zu verbergen versucht; es wäre auch aussichtslos gewesen.

in der halbleeren lufthansamaschine begrüsst mich eine sehr

freundliche stewardess. an ihrer bluse ein button: 
«wir sind jeden tag ausländer».

Notizen (unveröffentlicht)

Sich als Gast fühlen in zwei so unterschiedlichen Kulturen ist eine Erfahrung, die der

Dichter SAID zu nutzen weiß.

deutschland bietet ... hohlräume; die deutsche sprache eine neue haut.

deutschland: seine art, fremd zu sein. deutsch, die sprache seiner fremdheit, seiner

freiheit

wer, wenn ich schrie (unveröffentlicht)

SAID ist ein sehr vielseitiger Autor, der permanent nach neuen Ausdrucksmöglichkeiten sucht. Gedichte, Prosa, Essays, Theaterstücke, Hörspiele, erotische Texte, Arbeit mit dem Bild, Fotos und Gemälden. Auch phantastische Texte, die Grenzen sprengen und parallele Welten ins Leben rufen, mit anderen, manchmal gefährlichen, manchmal traumhaften Spielregeln, anderen Gesetzen. Offene Welten, offene Texte. Die Figuren Murmelstein, die Allegorie des Todes in „er wird kommen“ (beide unveröffentlicht) befremden. Diese Welten haben fließende Grenzen, das Erzählte ist oft widernatürlich.

Der Erzähler aber nie. Denn es geht immer wieder um die für ihn wichtigsten Themen: Exil als Schmerz, der nie zu überwinden ist, Kindheit als Utopie, Liebe als Lebenshilfe, Gleichgewicht zum Verlust, auch universelle Liebe des Menschen zum Menschen, die notwendig ist, um als Mensch zu bestehen, vor sich selbst und vor den anderen.

Fragen nach einem Sinn werden gestellt, in den Gedichten und in der Prosa, in manchmal sehr innovativer Form. Das geht vom Wunderbaren bis zum Grotesken. Über Traum und Utopie.

Wenn der Traum die Grenzen des Privaten überschreitet, wird er zur Kunst (3).

Bewegung zwischen dem Fremden und dem Eigenen - aufeinanderbezogen, auseinandergehalten. Aber sie bereichern sich gegenseitig.

Ich habe immer gelebt

von der Patina vergangener Zeiten

und den Samttönen kommender Morgen.

Selbstbildnis für eine ferne Mutter S. 55

Also doch Ent-fremdung? Einsicht, dass es Heimat vielleicht gar nicht gibt, dass man sie nur in sich selber trägt. Die Heimat ist für den Dichter die Sprache, in der er schreibt.

Meine eigentliche Heimstätte

ist die deutsche Sprache,

die mir Zuflucht geboten hat.

Der lange Arm der Mullahs S. 136

als ich 17 war, wünschte ich mir, daß das deutsche ein teil von mir würde. heute bin

ich 56 und weiß, daß ich ein teil dieser sprache bin. das ist viel schöner.

In Deutschland leben S. 28

Grenzen wurden also nicht nur überschritten, sondern auch überwunden, ohne die zwei Kulturen gegeneinander auszuspielen. Der Weg, den SAID gelaufen ist, ist der Weg zur Freiheit. Zu seiner eigenen Freiheit. Zu sich selbst. Nach und nach kam das Gefühl, das Recht zu haben, ein Fremder zu sein und es auch bleiben zu dürfen. Denn sind wir nicht alle überall Fremde? Bedeutet dieses Außenseitertum nicht auch Öffnung? Heimat ist überall oder nirgends.

Durch die Literatur wird die nationale Zugehörigkeit aufgehoben. 


\section{BIBLIOGRAPHIE}

Wunder und Dialektik haben miteinander zu tun.

Das Bindeglied ist die Zeit (3)!

\section{Bibliographie:}

liebesgedichte. P. Kirchheim, München 1981

wo ich sterbe, ist meine fremde. Gedichte P. Kirchheim, München 1984

ich und der schah. die beichte des ayatollah. hörspiele. Perspol-Verlag, Hamburg 1987

dann schreie ich, bis stille ist. Gedichte. Heliopolis, Tübingen 1990

selbstbildnis für eine ferne mutter. ein poem. P. Kirchheim, München 1992

der lange arm der mullahs. notizen aus meinem exil. C.H. Beck, München 1995

es war einmal eine blume. ein märchen mit bildern von kveta pacovska. Salzburg 1998

sei nacht zu mir. liebesgedichte. C.H. Beck, München 1998

die ballade vom esel trauermaul. ottensheim an der donau 1999

dieses tier, das es nicht gibt. Ein bestiarium. C.H. Beck München 1999

landschaften einer fernen mutter. C.H. Beck München 2001

clara. ein märchen mit bildern von moidi kretschmann. st. pölten 2001

außenhaut binnenträume. Neue gedichte. C.H. Beck, München 2002

friedrich hölderlin empfängt niemanden mehr. hörbuch. C.H. Beck, München 2002 (cd)

in deutschland leben. Ein gespräch. C.H. Beck, München 2004

auf den leib. Mit Fotos von james dummler. GeraNova/Bruckmann, München 2004

ich und der islam. C.H. Beck, München 2005

\section{Hörspiele:}

„wo ich sterbe, ist meine fremde“, „Sender freies Berlin“ 1981, mit der stimme von Paul Burian (Schaubühne).

„ich und der schah“, „Sender freies Berlin“ 1982, mit den Stimmen von Udo Samel und Armin Müller-Stahl.

„die beichte des ayatollah“, „bayerischer Rundfunk“ 1984, mit der Stimme von Wolfgang Büttner.

„landschaften einer mutter“, Koproduktion „,norddeutscher Rundfunk“ und „Südwestfunk“ 1996.

„sir alfred exterritorial“, „Südwestfunk“ 1997,

“friedrich hölderlin empfängt niemanden mehr"Regie: Hans Gerd Krogmann, Produktion: SWR 2001 


\section{Auf Französisch erschienen sind bis jetzt :}

« La fleur sans couleur » Conte illustré par Kveta Pacovska, Éditions Nord-Sud, Paris 1998

« Paysages d'une Mère lointaine » Éditions Métailié, paris 2003

\section{Literarische Preise :}

1986 Literaturpreis der Stadt München

1992 Civis-Hörfunkpreis

1994 premio letterario internazionale „Jean Monnet“

1996 Preis der Stadt Heidelberg „Literatur im Exil“

1997 Stipendium „villa aurora“, Los Angeles, USA

1997 Hermann-Kesten-Medaille des Pen-Zentrums Deutschland

1998 literaturstipendium der landeshauptstadt graz

2002 Adelbert-von-Chamisso-Preis

2005-2006 Stipendium «Mont Noir» Saint-Jans-Cappel Frankreich

2006 Goethe-Medaille

Zwischen 2000 et 2002 Präsident des deutschen PEN Clubs.

\section{NOTES}

1. Die allerersten Werke ausgenommen schreibt SAID immer alles klein.

2. Einige Zitate, auch etliche Gedichte sind zurzeit unveröffentlicht. Der Schriftsteller hat sie mir freundlicherweise zukommen lassen.

3. In einer Mail.

\section{RÉSUMÉS}

SAID 1947 in Teheran geboren, kommt 1965 nach Deutschland, um zu studieren. Er nimmt an der Studentenbewegung 1968 teil, eine Bewegung, die sich, insbesondere in München, gegen den Schah richtet, der zu dieser Zeit auf Staatsbesuch ist. SAID engagiert sich in der oppositionellen Studentenorganisation, lernt aber in jenen Jahren ganz intensiv die deutsche Sprache, die ihn bis heute noch fasziniert. Er fängt auch bald zu schreiben an, veröffentlicht schon 1982 seinen ersten Gedichtband. 1979 kehrt er nach Teheran zurück, muss aber feststellen, dass er in der Heimat so fremd wie in Deutschland ist, und dass es für ihn in der Republik der Mollahs kein Zurück mehr gibt. SAID hat Gedichte, Prosa, Kindergeschichten, erotische Texte und politische Essays geschrieben. Sehr verschiedene Gattungen, jedoch werden drei Themen immer wieder aufgegriffen: der Schmerz des Exils, die Verfolgung der Dissidenten, die Kindheitserinnerungen 
und die Liebe als Zuflucht. In seinen politischen Texten setzt sich SAID für Toleranz und das Nebeneinander der Weltanschauungen ein - während er doch, diskret aber entschieden, dem Westen einen Spiegel vor die Nase hält. Er versucht zwischen Orient und Okzident zu vermitteln und meistert diese Dynamik durch die Sprache, eine knappe, präzise Sprache, die eine ganz besondere Beleuchtung der Dinge bewirkt, eine Beleuchtung, die gerade dadurch glaubhaft ist, dass sie Schatten aufkommen lässt. SAIDs Weg in der Sprache ist Dynamik zu sich selbst.

SAID, né à Téhéran en 1947, arrive en Allemagne en 1965 pour y faire des études. Il participe à la révolte des étudiants de 68, une révolte à laquelle s'ajoute, surtout à Munich, une vive opposition au Schah en visite officielle à ce moment-là. SAID milite dans l'organisation des étudiants dissidents, mais s'emploie surtout à apprendre l'allemand qui exerce sur lui une fascination qui ne se démentira jamais. Il prend rapidement la plume et publie son premier recueil de poésie en 1982. Il retourne à Téhéran en 1979, mais s'aperçoit qu'un retour au pays serait vain, voire dangereux. Il n'y a pas d'avenir dans la République des Mollahs. SAID écrit une œuvre variée en langue allemande, poésie, prose, contes pour enfants, contes fantastiques, petits textes érotiques, essais politiques. Les trois axes sont invariablement la souffrance de l'exil, les persécutions dues à la dissidence ou à l'altérité, les souvenirs d'enfance et l'amour comme refuge. Dans son œuvre politique, il prône la tolérance et la diversité, tout en tendant discrètement un miroir aux sociétés occidentales. SAID est un pont entre deux univers qu'il cherche à dompter par une langue, précise et concise, un éclairage particulier des événements qui dévoile l'essentiel par les ombres qu'ils projettent. Le cheminement de SAID dans l'écriture est une quête de lui-même, de son être dans le monde.

\section{INDEX}

Mots-clés : Iran, émigration iranienne, littérature de la migration, exil, altérité

\section{AUTEURS}

\section{FRANCINE ROUBY}

Lycée - Khâgne - Douai 